\title{
Estimating the temporal precision and size of correlated groups of neurons from population activity Sebastien Louis* and Sonja Grün
}

\author{
Address: Theoretical Neuroscience Group, RIKEN Brain Science Institute, 2-1 Hirosawa, Wako-shi, 351-0198 Saitama, Japan \\ Email: Sebastien Louis* - sgr-louis@brain.riken.jp \\ * Corresponding author
}

from Eighteenth Annual Computational Neuroscience Meeting: CNS*2009

Berlin, Germany. 18-23 July 2009

Published: 13 July 2009

BMC Neuroscience 2009, I0(SuppI I):P253 doi:I0.II86/I47I-2202-I0-SI-P253

This abstract is available from: http://www.biomedcentral.com/I47I-2202/I0/SI/P253

(c) 2009 Louis and Grün; licensee BioMed Central Ltd.

\section{Introduction}

The efficient detection of higher order correlations in massively parallel data is of great importance and represents a significant challenge. To overcome the combinatorial explosion of different spike patterns taking place as the number of neurons increase, a method based on population measures would prove very useful. Following previous work in this direction $[1,2]$, we examine the distribution of spike counts across neurons per bin (complexity distribution) to extract the size of correlated groups of neurons and their temporal precision.

\section{Results}

To begin, we provide general analytical approximations to the complexity distribution for a known generation process [3] which we extend to allow for multiple correlated groups of neurons. Synchronous events spanning the groups are generated at spike times of a Bernoulli mother process proper to each group. Both the probability $(p)$ and the precision (jitter $j$ ) with which the spikes are copied over from the mother process can be modulated. The synchronous events are then superimposed onto background single neuron Bernoulli processes of time resolution $h=1$ ms. Lastly, the parallel spike trains are binned on a larger time resolution $w$ and the spike counts summed across the neurons. For this generation process we obtain a relatively simple analytical prediction for the complexity distribution in the case that the synchronous groups are non-overlapping, for arbitrary firing rates, jitter and bin width. The validity of our analytical predictions was verified through simulations, exploring different bin width and jitter configurations. An example is given in figure 1(a), where two disjoint synchronous groups of 5 and 15 neurons are active within a total population of 30 neurons $(j= \pm 3 \mathrm{~ms}$ and $w=10 \mathrm{~ms}$ ). The blue markers indicate the simulation results, the red line the predicted distribution and the green dotted line the expected distribution assuming independence of the neurons. Taking the difference between the predicted and the expected distributions yields a bimodal function (cyan curve in (a)). By following the position of the second maximum, reflecting the presence of higher complexity components caused by synchrony, through different bin widths, we were able to correctly infer the size of a single synchronous group present in the data, along with its jitter. This is done by locating the change in slope of the position of the second maximum, as shown in figure 1(b), where a synchronous group of 30 neurons out of 50 is active $(j= \pm 3 \mathrm{~ms})$. The change in slope is located at the green circle, whose center coordinates are $7 \mathrm{~ms}(2|j|+1)$ along the vertical axis and 30 along the complexity axis, providing a correct estimate of the actual parameters used.

\section{Conclusion}

The results presented in this work point to a rapid method for estimating the presence or not, and even the order as well as the temporal precision of a single synchronous group contained in a large population of neurons without going through pattern analysis. 
(a)
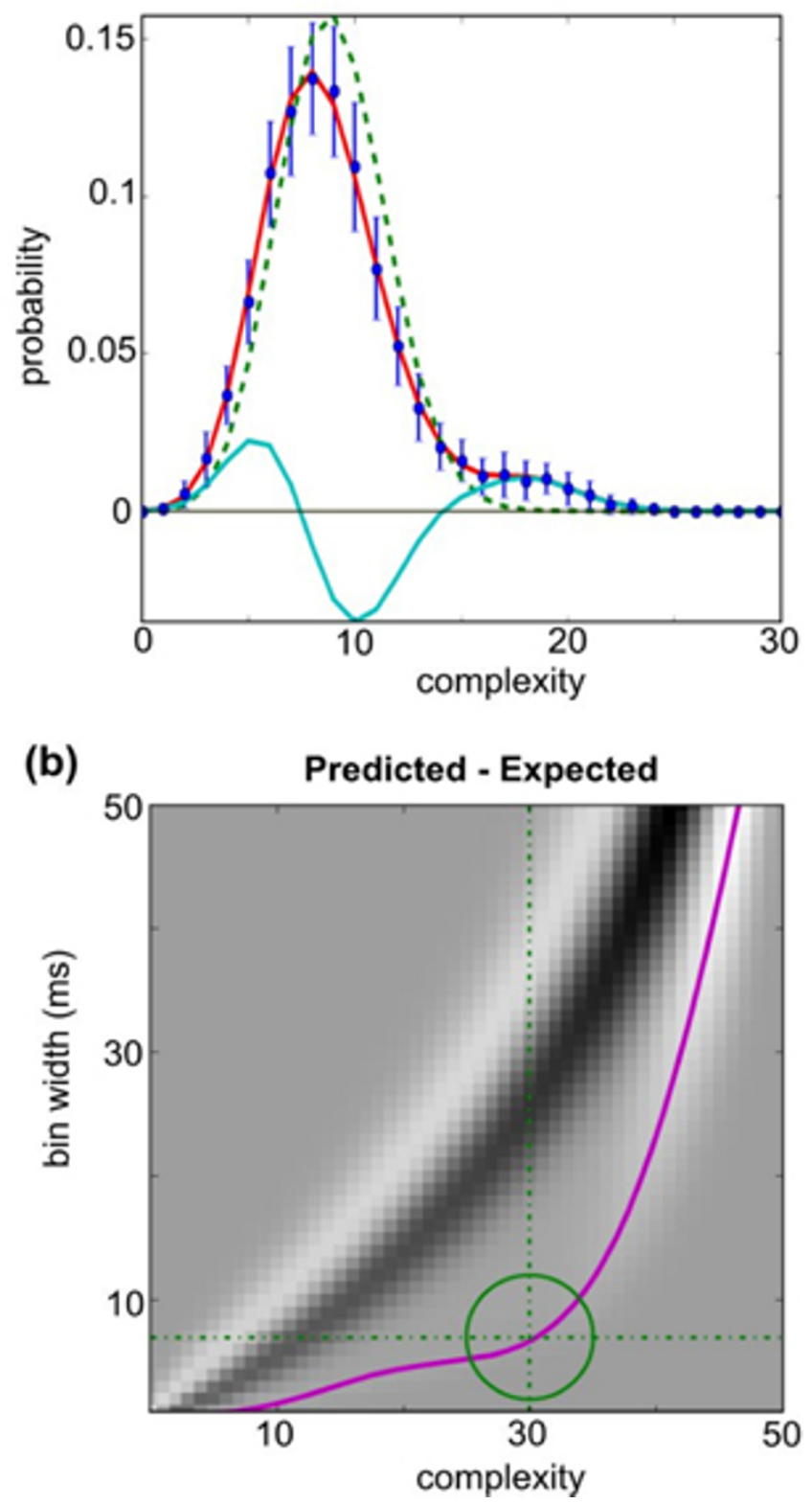

Figure I

\section{References}

I. Grün S, Abeles M, Diesmann M: Impact of higher-order correlations on coincidence distributions of massively parallel data. Lecture Notes in Computer Science, "Dynamic Brain - from Neural Spikes to Behaviors" 2008, 5286:96-II4.

2. Staude B, Rotter S, Grün S: CuBIC: cumulant based inference of higher-order correlations in massively parallel spike trains. 2008 in press.

3. Kuhn A, Aertsen A, Rotter S: Higher-order statistics of input ensembles and the response of simple model neurons. Neural Comput 2003, 15:67-101.
Publish with Biomed Central and every scientist can read your work free of charge

"BioMed Central will be the most significant development for disseminating the results of biomedical research in our lifetime. "

Sir Paul Nurse, Cancer Research UK

Your research papers will be:

- available free of charge to the entire biomedical community

- peer reviewed and published immediately upon acceptance

- cited in PubMed and archived on PubMed Central

- yours - you keep the copyright 